\title{
Cooling of Sr to high phase-space density by laser and sympathetic cooling in isotopic mixtures
}

\author{
G. Ferrari, R. E. Drullinger, N. Poli, F. Sorrentino, and G. M. Tino* \\ Dipartimento di Fisica and LENS, Istituto Nazionale Fisica Nucleare, Istituto Nazionale Fisica della Materia, \\ Polo Scientifico-Università di Firenze, 50019 Sesto Fiorentino, Italy
}

(Received 19 July 2005; published 10 February 2006)

\begin{abstract}
Based on an experimental study of two-body and three-body collisions in ultracold strontium samples, a novel optical-sympathetic cooling method in isotopic mixtures is demonstrated. Without evaporative cooling, a phase-space density of $6 \times 10^{-2}$ is obtained with a high spatial density that should allow us to overcome the difficulties encountered so far to reach quantum degeneracy for $\mathrm{Sr}$ atoms.
\end{abstract}

DOI: 10.1103/PhysRevA.73.023408

PACS number(s): 32.80.Pj, 05.30.Jp, 32.80.-t., 34.50.-s

The combination of laser cooling [1] and evaporative cooling has led to reach ultralow temperatures and to observe Bose-Einstein condensation (BEC) and Fermi degeneracy for different atoms [2]. Laser cooling is indeed very effective to reach phase-space densities within a few orders of magnitude of quantum degeneracy. The limits in cooling at high density are set by the optical depth of the sample, i.e., reabsorption of scattered light, and light-assisted atom-atom collisions. Forced evaporative cooling [3] represents the common way to circumvent these limits. However, this procedure does not work for all atoms. In particular, among the atoms cooled with optical methods, none of the alkali-earth atoms reached quantum degeneracy so far, except ytterbium which has a similar electronic structure [4]. A phase-space density of $\sim 10^{-1}$ was reported for $\mathrm{Sr}[5]$ but BEC could not be reached. $\mathrm{Sr}$ is of interest for several reasons: In a natural strontium sample, different isotopes can be investigated with different statistics; ${ }^{88} \mathrm{Sr}(82.6 \%),{ }^{86} \mathrm{Sr}(9.8 \%)$, and ${ }^{84} \mathrm{Sr}(0.6 \%)$ are bosons with zero nuclear spin; ${ }^{87} \mathrm{Sr}(7 \%)$ is a fermion with nuclear spin $I=9 / 2$. A BEC of $\mathrm{Sr}$ atoms would allow the study of 0 -spin condensates, fast optical cooling and continuous coherent matter-wave sources, quantum devices both for frequency standards and inertial sensors. Besides the studies towards quantum degeneracy, Sr has recently been the subject of active research in various fields spanning from laser cooling physics $[6,7]$, investigation of ultranarrow transitions towards future optical clocks [8-11], multiple scattering [12], and collisional physics [13-17].

In this article, we present a scheme for the production of a high phase-space density sample of atomic strontium. Since collisional processes play a crucial role and relevant parameters are not well known for this atom, we studied 2-body and 3-body collisions both in pure samples, and isotopic mixtures. Our results show that usual evaporative cooling methods are difficult to apply in a single species sample of ${ }^{86} \mathrm{Sr}$ or ${ }^{88} \mathrm{Sr}$. The investigation of an ultracold ${ }^{86} \mathrm{Sr}-{ }^{88} \mathrm{Sr}$ mixture revealed a large interspecies elastic cross section; this allowed us to demonstrate an optical-sympathetic cooling method conceptually different from what was done so far on neutral atoms $[18,19]$ and somehow analogous to the one used for trapped ions [20], achieving a phase-space density

*Electronic address: Guglielmo.Tino@fi.infn.it of $6 \times 10^{-2}$ in conditions suitable to further cooling of the sample towards BEC.

The experimental setup, based on the apparatus previously described in [8,21], allows us to trap single-isotope samples or isotopic mixtures of ${ }^{86} \mathrm{Sr}$ and ${ }^{88} \mathrm{Sr}$ atoms in an optical dipole trap. The loading procedure starts with a magneto-optical trap on the ${ }^{1} S_{0^{-}}{ }^{1} P_{1}$ resonance transition at $461 \mathrm{~nm}$. During this phase, a decay channel from the excited state results in the accumulation of atoms in the magnetically trapped metastable ${ }^{3} P_{2}$ state [22]. The isotopic mixture is produced in the magnetic trap by stepping the frequency of the trapping laser to the appropriate values. After loading the magnetic trap, atoms are optically pumped back into the electronic ground state and a second cooling stage is applied based on a magneto-optical trap operating on the narrow ${ }^{1} S_{0^{-}}{ }^{3} P_{1}$ transition at $689 \mathrm{~nm}$. At this point we typically obtain $10^{7}{ }^{88} \mathrm{Sr}$ atoms at $2 \mu \mathrm{K}$, and $10^{6}{ }^{86} \mathrm{Sr}$ atoms at $1 \mu \mathrm{K}$. The isotopic mixture is then loaded into an optical dipole trap by superposing a focused $922 \mathrm{~nm}$ laser on the red magneto-optical trap, resulting in a $90 \mu \mathrm{K}$ deep potential with radial and axial frequencies of $2 \mathrm{kHz}$ and $26 \mathrm{~Hz}$, respectively. To optimize the transfer efficiency from the red magneto-optical trap into the optical dipole trap, the optical dipole beam waist is axially displaced from the magnetooptical trap by $500 \mu \mathrm{m}$ (corresponding to $2 / 3$ of the dipole beam Rayleigh range and two times the red magneto-optical trap radii) in order to increase the magneto-optical trapdipole trap spatial overlap [23]. The measured 6 s lifetime in the optical trap is consistent with the residual background gas pressure in our apparatus. The off-resonant photon scattering induces a heating of $0.4 \mu \mathrm{K} / \mathrm{s}$. The polarization of the dipole beam is set to nearly fulfill the requirements for trapping at the "magic wavelength" that cancels the differential Stark shift for the ${ }^{1} S_{0^{-}}{ }^{3} P_{1}$ transition [24]. The atom number and temperature are measured independently on the two isotopes by absorption imaging with a resonant probe beam, and the contribution of the nonresonant isotope is taken into account. The spatial density is inferred from the measured temperature and the trapping potential.

Two-body and three-body collisions are studied by loading single species or the isotopic mixture into the optical dipole trap. For the investigation of 2-body elastic collisions, the gas is initially put out of equilibrium by a sudden perturbation of the optical potential (pure samples), or by an 


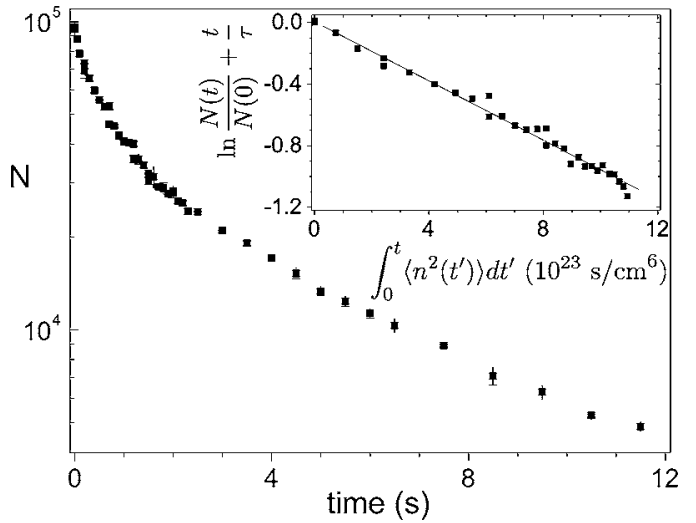

FIG. 1. Nonexponential decay of the number of ${ }^{86} \mathrm{Sr}$, trapped in the optical dipole trap due to 3-body recombination. Inset: the 3-body recombination rate constant is given by the slope of the natural log of the atom number with respect to $\int_{0}^{t}\left\langle n^{2}\left(t^{\prime}\right)\right\rangle d t^{\prime}$ where $\left\langle n^{2}\left(t^{\prime}\right)\right\rangle=1 / N\left(t^{\prime}\right) \int_{V} n^{3}\left(\vec{r}, t^{\prime}\right) d^{3} r$ (see the text). The term $t / \tau$ accounts for losses due to background collisions.

isotope-selective laser cooling pulse (mixture). We relate the measured thermalization time at the given density and temperature to the elastic cross section $\sigma$ following and generalizing the approach described in [25]. The resulting values are $\sigma_{88-88}=(3 \pm 1) \times 10^{-17} \mathrm{~m}^{2}, \sigma_{86-86}=(1.3 \pm 0.5) \times 10^{-14} \mathrm{~m}^{2}$, and $\sigma_{86-88}=(4 \pm 1) \times 10^{-16} \mathrm{~m}^{2}$. In the single isotope case, the results are consistent with the corresponding scattering lengths measured by photoassociation spectroscopy $[16,17]$. No previous reference data is available for the two-isotope collisions.

We studied the 3-body recombination rate $K_{3}$ by loading a single isotope (either ${ }^{86} \mathrm{Sr}$ or ${ }^{88} \mathrm{Sr}$ ) into the dipole trap and observing the evolution of the atom number and temperature. With ${ }^{88} \mathrm{Sr}$ we found no experimental evidence for nonexponential decay and, given the initial atom density at the trap center of $3 \times 10^{13} \mathrm{~cm}^{-3}$, we set an upper bound on $K_{3}^{88}$ at $10^{-27} \mathrm{~cm}^{6} / \mathrm{s}$. To determine $K_{3}^{86}$ we followed the analysis described in [26]: the loss rate due to the 3-body recombination is modeled by the rate equation

$$
\frac{d N}{d t}=-K_{3} \int_{V} n^{3}(\vec{r}, t) d^{3} r
$$

or equivalently

$$
\ln \frac{N(t)}{N(0)}=-K_{3} \int_{0}^{t} d t^{\prime} \int_{V} \frac{n^{3}\left(\vec{r}, t^{\prime}\right)}{N\left(t^{\prime}\right)} d^{3} r
$$

where $n$ is the density, and $N(t)$ is the atom number at time $t$. Figure 1 shows the data and the fit using Eq. (2) from which we obtain the rate constant $K_{3}^{86}=(1.0 \pm 0.5) \times 10^{-24} \mathrm{~cm}^{6} / \mathrm{s}$. This recombination rate is more than three orders of magnitude larger than the typical values for ultracold alkali vapors [27].

The first consequence of these results is that evaporative cooling on pure samples of either ${ }^{86} \mathrm{Sr}$ or ${ }^{88} \mathrm{Sr}$ cannot be efficient. ${ }^{86} \mathrm{Sr}$ presents an extremely large elastic cross section, a good point to establish a fast thermalization, but the 3 -body recombination rate introduces a loss channel that is fatal with the typical geometries accessible through optical dipole trapping. An optical trap with a much larger trapping volume would partially suppress this loss channel [28]. ${ }^{88} \mathrm{Sr}$ instead turns out to be stable against 3-body decay, but the small elastic cross-section results in a long thermalization time compared to typical trap lifetime. Indeed, in our experiment evaporative cooling of ${ }^{88} \mathrm{Sr}$ by reducing the dipole beam intensity allowed us to increase the phase-space density to a maximum of $2 \times 10^{-1}$, limited by the reduction of the thermalization rate during the evaporation and by the background-limited lifetime $\tau=6 \mathrm{~s}$.

These results led to a new all-optical sympathetic cooling scheme. The basic idea of the new cooling method is to use a mixture of isotopes trapped in the dipole trap: one isotope is laser cooled and by elastic collisions cools the other isotope which is the one of interest. The advantage, compared to the schemes used so far for neutral atoms, is that the limitations of laser cooling of spatially dense and optically thick samples are drastically reduced. In this work, continuous laser cooling of ${ }^{86} \mathrm{Sr}$ leads to heat dissipation in a ${ }^{88} \mathrm{Sr}$ sample by sympathetic cooling; the small ${ }^{86} \mathrm{Sr}$ optical depth does not limit the achievable minimum temperature. Sympathetic cooling with neutral atoms normally requires a thermal bath with a large heat capacity with respect to that of the sample to be cooled. This is due to the fact that when the thermal bath is cooled by evaporative cooling, each lost atom carries an energy of the order of few times the temperature of the sample. In the case of optical-sympathetic cooling, each laser-cooled atom can subtract an energy of the order of the optical recoil in a time corresponding to a few lifetimes of the excited state (say 4 , when operating at $1 / 4 I_{\text {sat }}$ ), without being lost. Considering the ${ }^{1} S_{0^{-}}{ }^{3} P_{1}$ transition, the power subtracted per atom is of the order of $6 \mathrm{mK} / \mathrm{s}$; if the interisotope thermalization is fast enough, each ${ }^{86} \mathrm{Sr}$ atom can cool about $10^{3}{ }^{88} \mathrm{Sr}$ atoms down to $\mu \mathrm{K}$ temperatures in $1 \mathrm{~s}$. In the ${ }^{86} \mathrm{Sr}$ ${ }^{88} \mathrm{Sr}$ isotope mixture the relatively large interspecies crosssection results in thermalization times typically of the order of few milliseconds. This thermalization is fast even on the time scale of laser cooling on the intercombination ${ }^{1} S_{0^{-}}{ }^{3} P_{1}$ transition. On the other hand, the $164 \mathrm{MHz}{ }^{86} \mathrm{Sr}^{88}{ }^{8} \mathrm{Sr}$ isotopic shift and the natural linewidth of $7.6 \mathrm{kHz}$ for the ${ }^{1} S_{0^{-}}{ }^{3} P_{1}$ transition, makes laser cooling on one isotope insensitive to the presence of the other.

We implemented the optical-sympathetic cooling scheme by extending the temporal overlap between the optical dipole trap and the ${ }^{86} \mathrm{Sr}$ red magneto-optical trap after switching off the ${ }^{88} \mathrm{Sr}$ magneto-optical trap. During this phase the parameters that optimize the ${ }^{88} \mathrm{Sr}$ cooling are substantially the same as those typically employed in the second cooling stage (intensity $30-100 I_{\mathrm{sat}}$ with $I_{\mathrm{sat}}=3 \mu \mathrm{W} / \mathrm{cm}^{2},-200 \mathrm{kHz}$ frequency detuning, magnetic field gradient $0.6 \mathrm{mT} / \mathrm{cm})$. The horizontal displacement between the red magneto-optical trap and the dipole trap optimizes the spatial overlap and provides a continuous flux of ${ }^{86} \mathrm{Sr}$ atoms from the magnetooptical trap to the optical dipole trap replacing the atoms that are lost during the optical-sympathetic cooling due to light 


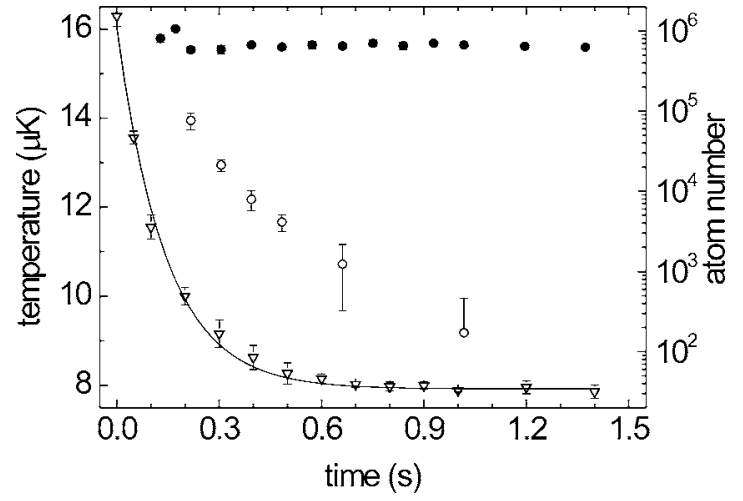

FIG. 2. Dynamics of an optically trapped ${ }^{88} \mathrm{Sr}$ cloud sympathetically cooled with laser cooled ${ }^{86} \mathrm{Sr}$. Filled circles 0 : ${ }^{88} \mathrm{Sr}$ atom number. Open circles $\bigcirc:{ }^{86} \mathrm{Sr}$ atom number. The number of ${ }^{88} \mathrm{Sr}$ atoms remains constant during the process, while ${ }^{86} \mathrm{Sr}$ decays exponentially with a $80 \mathrm{~ms}$ time constant. Under optimized conditions, the temperature (triangles $\nabla$ ) decreases with a $150 \mathrm{~ms}$ time constant and the mixture is always at thermal equilibrium.

assisted collisions. Figure 2 reports the dynamics of opticalsympathetic cooling, starting from an initial temperature of $16 \mu \mathrm{K}$, limited by density dependent heating $[21,29]$. It can be observed that the cooling process does not induce losses on ${ }^{88} \mathrm{Sr}$ while the number of ${ }^{86} \mathrm{Sr}$ atoms exponentially decays with a $80 \mathrm{~ms}$ lifetime. About $100 \mathrm{~ms}$ after switching off the ${ }^{88} \mathrm{Sr}$ red magneto-optical trap, we observe that the mixture attains thermal equilibrium. From this point on the equilibrium is maintained, which is an essential condition for the implementation of this cooling scheme. Under optimized conditions (overall optical intensity $100 I_{\mathrm{sat}}$ ) the temperature decays from the initial value with a $150 \mathrm{~ms}$ time constant. The minimum attainable temperature depends both on the intensity of the ${ }^{86} \mathrm{Sr}$ cooling beam, and the ${ }^{88} \mathrm{Sr}$ density. Figure 3 reports the asymptotic temperature as a function of the overall cooling light intensity incident on the sample, for a fixed amount of trapped ${ }^{88} \mathrm{Sr}$. At high intensity, the ${ }^{86} \mathrm{Sr}$ lifetime increases due to the reduced spatial density and the final temperature is proportional to the intensity as expected from the usual Doppler theory. By lowering the intensity, the final

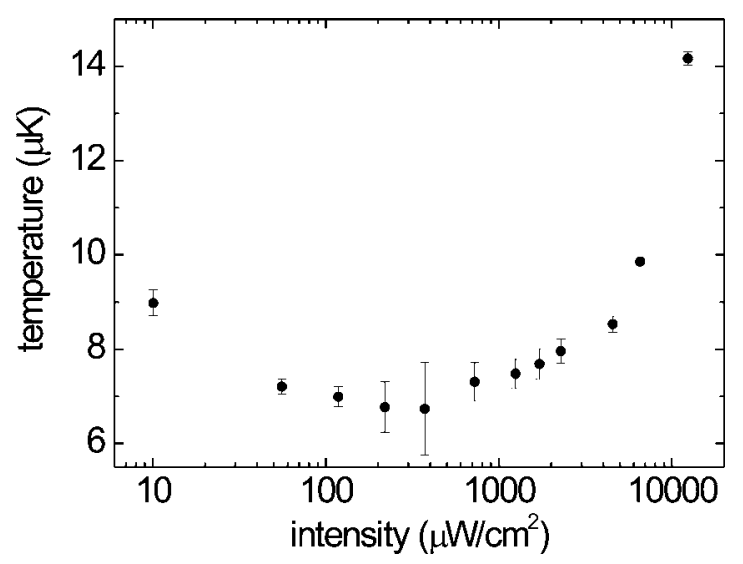

FIG. 3. Asymptotic temperature of the ${ }^{88} \mathrm{Sr}$ cloud for different intensities of the ${ }^{86} \mathrm{Sr}$ laser cooling beam on the ${ }^{1} S_{0^{-}}{ }^{3} P_{1}$ transition $\left(I_{\mathrm{sat}}=3 \mu \mathrm{W} / \mathrm{cm}^{2}\right)$. The ${ }^{88} \mathrm{Sr}$ atom number is $6 \times 10^{5}$.

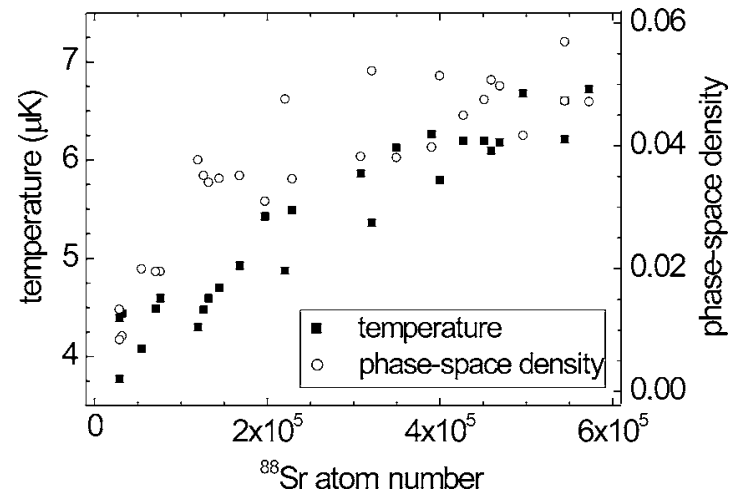

FIG. 4. Temperature and phase-space density of the ${ }^{88} \mathrm{Sr}$ cloud sympathetically cooled with laser cooled ${ }^{86} \mathrm{Sr}$, as a function of the ${ }^{88} \mathrm{Sr}$ atom number.

temperature and the ${ }^{86} \mathrm{Sr}$ lifetime decrease to the point where ${ }^{86} \mathrm{Sr}$ is lost too fast with respect to the cooling dynamics of ${ }^{88} \mathrm{Sr}$. The optimum values are obtained when the overall cooling light incident on the atoms has an intensity $\sim 30 I_{\text {sat }}$. For $6 \times 10^{5}{ }^{88} \mathrm{Sr}$ atoms trapped in the dipole trap, the final temperature is $6.7 \mu \mathrm{K}$ at a peak density of $1.3 \times 10^{14} \mathrm{~cm}^{3}$; the corresponding phase-space density is $5 \times 10^{-2}$. This value is only a factor of 2 lower with respect to what was previously obtained without forced evaporation [5], but it exhibits more favorable conditions for starting evaporative cooling, considering the higher spatial density (more than one order of magnitude higher) and the larger number of trapped atoms. By keeping the cooling parameters on ${ }^{86} \mathrm{Sr}$ fixed at the optimum value and by varying the number of trapped ${ }^{88} \mathrm{Sr}$, we determined the dependence of the final temperature with respect to the ${ }^{88} \mathrm{Sr}$ density. Figure 4 shows the dependence of ${ }^{88} \mathrm{Sr}$ temperature from the number of atoms in the trap. We determine the density-dependent heating $d T / d n$ $\simeq 2 \mu \mathrm{K} /\left(10^{14} \mathrm{~cm}^{-3}\right)$, which is 20 times lower than the equivalent value for pure laser cooled ${ }^{88} \mathrm{Sr}$ [29]. This strong reduction is a direct consequence of the optical distinction of the two isotopes on the ${ }^{1} S_{0^{-}}{ }^{3} P_{1}$ transition. The limit on the ${ }^{86} \mathrm{Sr}$ temperature of $4 \mu \mathrm{K}$ for zero ${ }^{88} \mathrm{Sr}$ density can be attributed to the laser cooling dynamics in the tightly confining potential of the dipole trap. Considering the power laws involved, reducing the optical dipole potential in the final stage of the optical-sympathetic cooling should lead to further cooling of the sample and considerably increase the ${ }^{88} \mathrm{Sr}$ phase-space density.

In conclusion, we investigated the collisional properties of ultracold $\mathrm{Sr}$ atoms of interest for the production of a BoseEinstein condensate. On pure ${ }^{86} \mathrm{Sr}$ we find a very large threebody recombination rate which sets stringent limits on the evaporation efficiency on this isotope. For ${ }^{88} \mathrm{Sr}$ we find that the small elastic cross section results in a long thermalization time compared to typical trap lifetime. This can explain why $\mathrm{BEC}$ of $\mathrm{Sr}$ was not achieved so far. On the other hand, for the ${ }^{86} \mathrm{Sr}-{ }^{88} \mathrm{Sr}$ mixture we measure a rather large interspecies elastic cross section. We exploit the fast ${ }^{86} \mathrm{Sr}-{ }^{88} \mathrm{Sr}$ thermalization to demonstrate an optical-sympathetic cooling scheme in which heat dissipation for one isotope is obtained by laser cooling; the sympathetically cooled component is more than 
one order of magnitude more abundant than the laser cooled one, reducing substantially the limitations associated to laser cooling at high spatial density. We cool up to $7 \times 10^{5}{ }^{88} \mathrm{Sr}$ atoms at a peak spatial density of $1.4 \times 10^{14} \mathrm{~cm}^{-3}$ and 6 $\times 10^{-2}$ phase space density. The combination of this cooling scheme to a final evaporative cooling stage on pure ${ }^{88} \mathrm{Sr}$ should allow to increase considerably the phase-space density opening the possibility to investigate quantum degenerate gases of Sr. An interesting possibility is also the development of new atomic sensors based on ultracold $\mathrm{Sr}$ atoms exploting the weak interatomic interaction and their small sensitivity to magnetic and electric fields.

We acknowledge fruitful discussions with M. Prevedelli and A. Simoni. We thank R. Ballerini, M. De Pas, M. Giuntini, and A. Hajeb for technical assistance. This work was supported by Agenzia Spaziale Italiana, Ente Cassa di Risparmio di Firenze, MIUR-COFIN 2002, Istituto Nazionale Fisica della Materia, Istituto Nazionale di Fisica Nucleare, and LENS.
[1] S. Chu, C. Cohen-Tannoudji, and W. D. Phillips, Rev. Mod. Phys. 70, 685 (1998).

[2] E. A. Cornell and C. E. Wieman, Rev. Mod. Phys. 74, 875 (2002); W. Ketterle, ibid. 74, 1131 (2002).

[3] H. F. Hess, Phys. Rev. B 34, 3476 (1986).

[4] Y. Takasu, K. Maki, K. Komori, T. Takano, K. Honda, M. Kumakura, T. Yabuzaki, and Y. Takahashi, Phys. Rev. Lett. 91, 040404 (2003).

[5] T. Ido, Y. Isoya, and H. Katori, Phys. Rev. A 61, 061403(R) (2000).

[6] X. Xu, T. H. Loftus, J. W. Dunn, C. H. Greene, J. L. Hall, A. Gallagher, and J. Ye, Phys. Rev. Lett. 90, 193002 (2003).

[7] T. H. Loftus, T. Ido, A. D. Ludlow, M. M. Boyd, and J. Ye, Phys. Rev. Lett. 93, 073003 (2004).

[8] G. Ferrari, P. Cancio, R. Drullinger, G. Giusfredi, N. Poli, M. Prevedelli, C. Toninelli, and G. M. Tino, Phys. Rev. Lett. 91, 243002 (2003).

[9] I. Courtillot, A. Quessada, R. P. Kovacich, A. Brusch, D. Kolker, J.-J. Zondy, G. D. Rovera, and P. Lemonde, Phys. Rev. A 68, 030501(R) (2003).

[10] M. Takamoto and H. Katori, Phys. Rev. Lett. 91, 223001 (2003).

[11] T. Ido, T. H. Loftus, M. M. Boyd, A. D. Ludlow, K. W. Holman, and J. Ye, Phys. Rev. Lett. 94, 153001 (2005).

[12] Y. Bidel, B. Klappauf, J. C. Bernard, D. Delande, G. Labeyrie, C. Miniatura, D. Wilkowski, and R. Kaiser, Phys. Rev. Lett. 88, 203902 (2002).

[13] A. Derevianko, S. G. Porsev, S. Kotochigova, E. Tiesinga, and P. S. Julienne, Phys. Rev. Lett. 90, 063002 (2003).

[14] R. Santra, K. V. Christ, and C. H. Greene, Phys. Rev. A 69, 042510 (2004), and references therein.

[15] R. Ciuryło, E. Tiesinga, and P. S. Julienne, Phys. Rev. A 71,
030701(R) (2005)

[16] M. Yasuda, T. Kishimoto, M. Takamoto, and H. Katori, Phys. Rev. A 73, 011403 (2006).

[17] P. G. Mickelson, Y. N. Martinez, A. D. Saenz, S. B. Nagel, Y. C. Chen, T. C. Killian, P. Pellegrini, and R. Côté, Phys. Rev. Lett. 95, 223002 (2005).

[18] C. J. Myatt, E. A. Burt, R. W. Ghrist, E. A. Cornell, and C. E. Wieman, Phys. Rev. Lett. 78, 586 (1997).

[19] F. Schreck, G. Ferrari, K. L. Corwin, J. Cubizolles, L. Khaykovich, M.-O. Mewes, and C. Salomon, Phys. Rev. A 64, 011402(R) (2001).

[20] R. E. Drullinger, D. J. Wineland, and J. C. Bergquist, Appl. Phys. 22, 365 (1980).

[21] N. Poli, R. E. Drullinger, G. Ferrari, J. Léonard, F. Sorrentino, and G. M. Tino, Phys. Rev. A 71, 061403(R) (2005).

[22] H. Katori, T. Ido, Y. Isoda, and M. Kuwata-Gonokami, in Atomic Physics 17, edited by E. Arimondo, P. de Natale, and M. Inguscio, AIP Conf. Proc. (AIP, New York, 2001), p. 382.

[23] S. J. M. Kuppens, K. L. Corwin, K. W. Miller, T. E. Chupp, and C. E. Wieman, Phys. Rev. A 62, 013406 (2000).

[24] T. Ido and H. Katori, Phys. Rev. Lett. 91, 053001 (2003).

[25] M. Arndt, M. Ben Dahan, D. Guéry-Odelin, M. W. Reynolds, and J. Dalibard, Phys. Rev. Lett. 79, 625 (1997).

[26] E. A. Burt, R. W. Ghrist, C. J. Myatt, M. J. Holland, E. A. Cornell, and C. E. Wieman, Phys. Rev. Lett. 79, 337 (1997).

[27] A. J. Moerdijk, H. M. J. M. Boesten, and B. J. Verhaar, Phys. Rev. A 53, 916 (1996).

[28] T. Weber, J. Herbig, M. Mark, H.-C. Naegerl, and R. Grimm, Science 299, 232 (2002).

[29] H. Katori, T. Ido, Y. Isoya, and M. Kuwata-Gonokami, Phys. Rev. Lett. 82, 1116 (1999). 